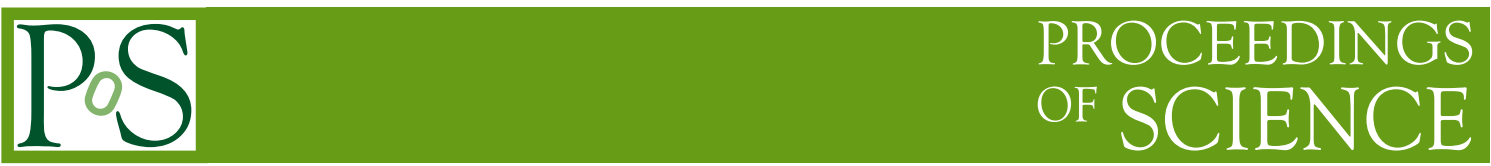

\title{
Inclusive jet measurements with the ATLAS experiment at the LHC
}

\author{
Peter $\operatorname{Loch}^{a, b, *}$, on behalf of the ATLAS Collaboration \\ ${ }^{a}$ Department of Physics, University of Arizona, Tucson, Arizona 85721, USA \\ ${ }^{b}$ CERN, Geneva, Switzerland
}

E-mail: Peter.Loch@cern.ch

The ATLAS experiment at the Large Hadron Collider (LHC) measures particle jet production not only in terms of cross sections but also to determine hadronic event shapes that represent the energy flow generated by hard parton emissions in the final state and to measure fundamental Standard Model parameters like the strong coupling constant $\alpha_{\mathrm{S}}$ in previously unexplored kinematic regimes. In particular the measurement of hadronic event shape variables with jets provides an important tool for testing the predictive power of hadronic final state generators with respect to the modelling of the complex energy flow patterns emerging from the proton-proton collisions at the LHC. The most recent results from ATLAS found that the tested generators generally have limited prediction power for the observed event shapes, with some models performing better for some of the shapes than others. The running of $\alpha_{\mathrm{S}}$ at high momentum transfers is extracted from measurements of the transverse energy-energy correlations between jets and the associated azimuthal asymmetries. The latest measurements performed by ATLAS extend the kinematics range well into the $\mathrm{TeV}$ regime.

40th International Conference on High Energy physics - ICHEP2020

July 28 - August 6, 2020

Prague, Czech Republic (virtual meeting)

\footnotetext{
${ }^{*}$ Speaker
} 


\section{Introduction}

The ATLAS experiment [1] at the Large Hadron Collider (LHC) at CERN, Geneva, Switzerland, provides a near full-coverage measurement of the final states emerging from the proton-proton collisions at $\sqrt{s}=13 \mathrm{TeV}$ (LHC Run 2). Important probes for the reconstruction of energy flow patterns in this final state are particle jets and their emission topologies which are largely driven by dynamics introduced by the strong force. The observed patterns are represented by a set of event shapes which are reconstructed in multijet final states. The theoretical expectation for these shapes can be calculated using quantum chromodynamics (QCD) at fixed order in terms of the strong coupling constant $\alpha_{\mathrm{S}}$. These calculations are matched with parton shower, fragmentation and hadronization models to form a generated final state of stable particles using various Monte Carlo (MC) generators. Event shapes constructed from jets clustered from these particles can then be compared to experimentally measured shapes using the same jet clustering algorithm on the experimental signals. This approach tests the prediction power of the used models. ATLAS conducted a comprehensive set of measurements of a set of meaningful event shapes at high precision [2] using the full LHC Run 2 dataset, corresponding to about $139 \mathrm{fb}^{-1}$. A summary of the most important findings from these measurements is presented in Sect. 2.

While the hadronic event shapes provide sensitive probes into the final state dynamics of hard emissions, the measurement of the transverse energy-energy correlations (TEEC) measured with jets is sensitive to the running of $\alpha_{\mathrm{S}}$ at the highest accessible momentum transfers. The recent evaluation of the TEEC and the associated azimuthal asymmetry (ATEEC) distributions in terms of a hard scale associated with the proton-proton collisions yielded a measurement of the running of $\alpha_{\mathrm{S}}$ well into the $\mathrm{TeV}$ region [3]. The most important results from these measurements are summarized in Sect. 3.

\section{Hadronic event shapes with jets}

Hadronic event shapes measured with jets provide proxies for several aspects of the hard energy flow emerging from the proton-proton collisions. The recent measurement conducted by ATLAS focusses on the highest emission scales by using jets with a transverse momentum of $p_{\mathrm{T}}>100 \mathrm{GeV}$. The jets are required to be within a pseudorapidity range of $|\eta| \leq 2.4$. This particular selection reduces background from pile-up jets. The jets are clustered using the anti- $k_{t}$ algorithm [4] provided by the FastJet [5] toolkit, with a distance parameter $R=0.4$. In the experiment, the jets are reconstructed from particle flow objects [6] at very high precision, with systematic uncertainties on the jet energy scale of $\lesssim 1 \%$ [7]. Only events with at least two jets in the selected phase space are considered for the measurement. The hardness of the interaction is measured by the scalar sum of the transverse momenta of the two leading jets, $H_{\mathrm{T} 2}=p_{\mathrm{T}}^{\text {lead }}+p_{\mathrm{T}}^{\text {sublead }}$. Only events with $H_{\mathrm{T} 2}>1 \mathrm{TeV}$ are considered for the analysis.

Particle jets generated in MC simulations are reconstructed in the same phase space. Fully simulated MC samples with particle and detector jets are used to determine the parameters needed to unfold the distributions from data to the particle level.

The six event shapes considered are commonly characterized by vanishing for back-to-back jet topologies in $2 \rightarrow 2$ QCD processes in the transverse plane. They are at maximum for the most 

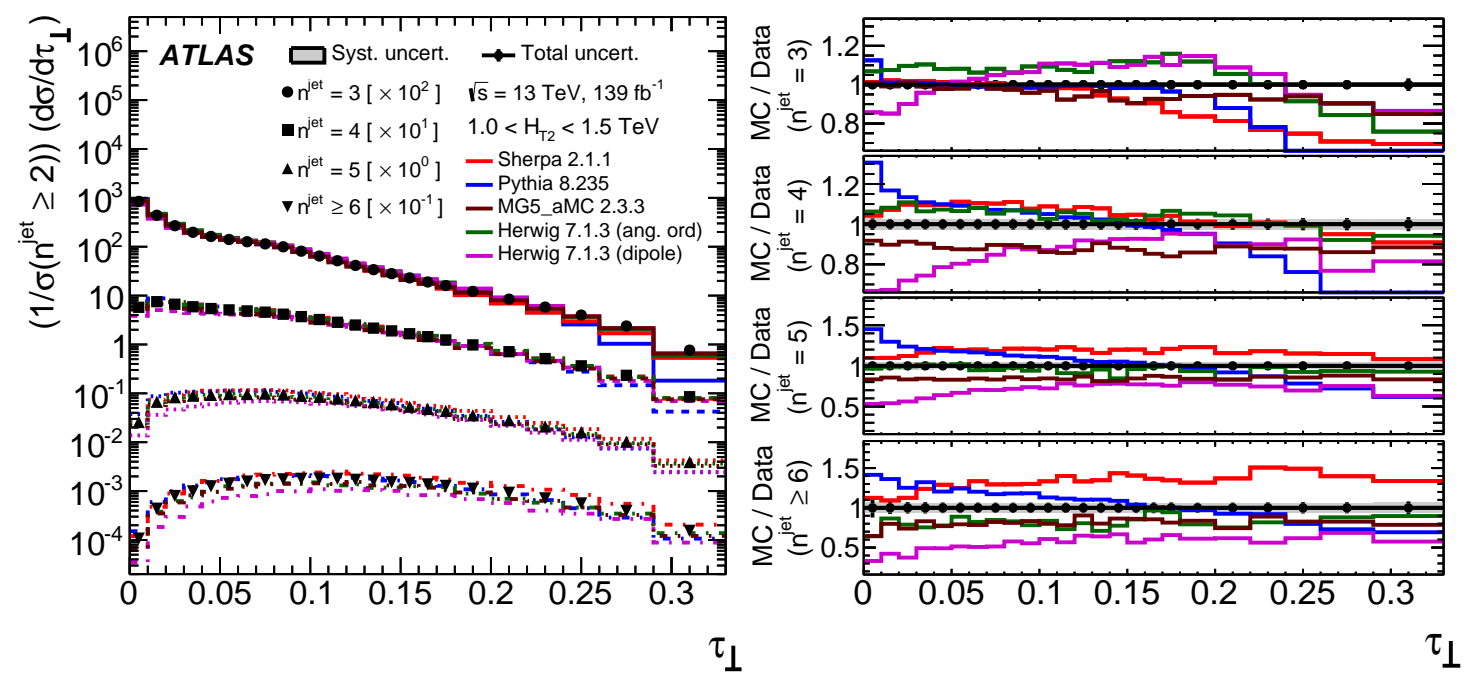

Figure 1: The normalised differential transverse thrust cross section, as given in Eq. 1 for $X=\tau_{\perp}$, for multijet production for $1 \mathrm{TeV}<H_{\mathrm{T} 2}<1.5 \mathrm{TeV}$ and various $n^{\text {jet }} \geq 3$ for data and MC simulations employing various physics models. The right panel shows the MC-to-data ratio of the cross sections for the different $n^{\text {jet }}$ selections (figure from Ref. [2]).

uniform transverse momentum distribution:

Transverse thrust $T_{\perp}$ and minor component $T_{m}$ :

$T_{\perp}$ is used to reconstruct $\tau_{\perp}=1-T_{\perp}$, where $\tau_{\perp} \rightarrow 0$ indicates uniform and $\tau_{\perp} \rightarrow 1-2 / \pi$ indicates back-to-back topologies in the transverse plane. $T_{m}$ is limited to $0 \leq T_{m}<2 / \pi$, with larger values indicating increased energy flow outside of plane spanned by thrust and beam axes.

Transverse sphericity $S_{\perp}$ :

$S_{\perp}$ is reconstructed from the eigenvalues of the transverse sphericity tensor $\mathcal{M}_{x y}$, with $0 \leq S_{\perp} \leq 1$ and lower values indicating back-to-back and higher values indicating isotropic energy flow in the transverse plane.

\section{Aplanarity A:}

$A$ is reconstructed from the eigenvalues of the sphericity tensor $\mathcal{M}_{x y z}$ and characterised by $0 \leq A \leq 1$ and higher values indicating a less planar event shape.

Multijet shape observables $C, D$ :

$C$ and $D$ are both reconstructed from eigenvalues of $\mathcal{M}_{x y z}$ such that $C=0$ for $n^{\text {jet }}<3$, where $n^{\text {jet }}$ is the number of jets in the event, and $0<C \leq 1$ for $n^{\text {jet }}>2$. The observable $D$ vanishes if all jet momenta lie in the same plane. Otherwise it assumes non-trivial values within $0<D \leq 1$.

The measurement is presented as the ratio of the differential cross section with respect to a given event shape variable $X$ to the fiducial cross section $\sigma\left(n^{\text {jet }} \geq 2\right)$, evaluated for various $\left(H_{\mathrm{T} 2}, n^{\text {jet }}\right)$ bins:

$$
\Sigma_{X}=\frac{1}{\sigma\left(n^{\mathrm{jet}} \geq 2\right)} \times \frac{d \sigma\left(H_{\mathrm{T} 2}, n^{\mathrm{jet}}\right)}{d X} \text {, with } X \in\left\{\tau_{\perp}, T_{m}, S_{\perp}, A, C, D\right\}
$$



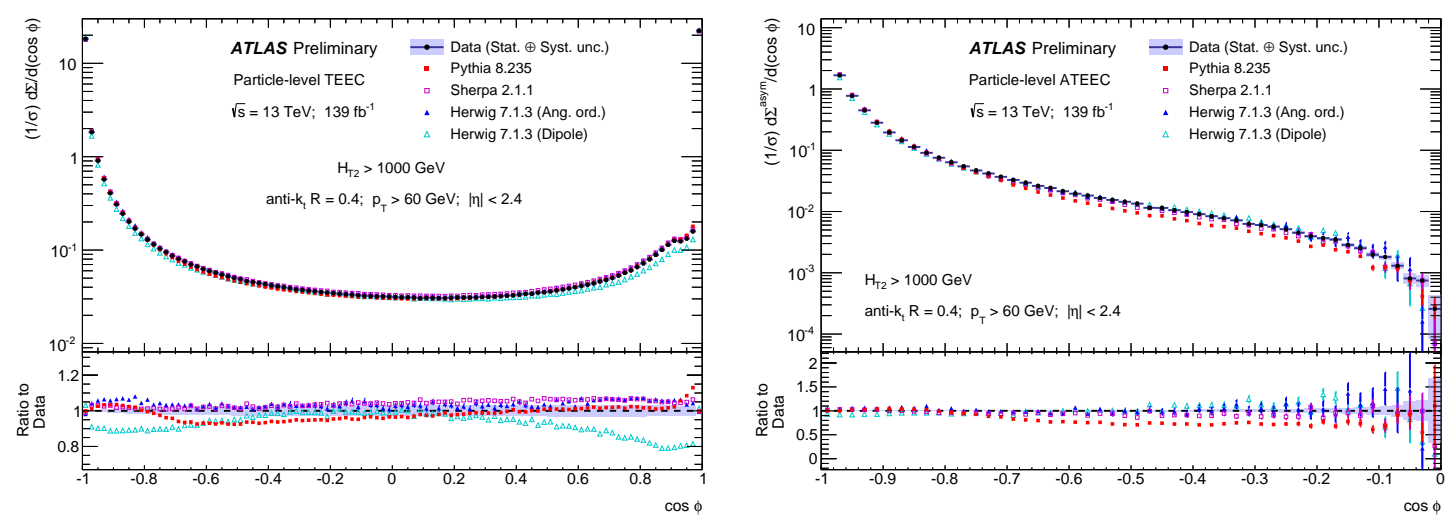

Figure 2: The TEEC (left figure) and ATECC (right figure) distributions measured with a multijet sample, unfolded to particle level and compared to several MC generators. The results shown are from the inclusive analysis $\left(H_{\mathrm{T} 2}>1 \mathrm{TeV}\right)$ (figures taken from Ref. [3]).

Figure 1 shows the result for unfolded data and various MC generators including PyTHIA 8.235 [10] with a $2 \rightarrow 2$ matrix element (ME) at leading order (LO) QCD, SHERPA 2.2.1 [11] with $2 \rightarrow\{2,3\}$ ME at LO accuracy, HeRwIG 7.1.3 [12,13] with angular ordered parton shower and with dipole parton shower at $2 \rightarrow 2$ next-to-leading order (NLO) accuracy and $2 \rightarrow 3$ at LO accuracy, and MADGRAPH5_AMC 2.3.3 [14] with $2 \rightarrow\{2,3,4\}$ at LO accuracy. A large spread in the generator modelling is observed in the comparison to data. Final states with $n^{\text {jet }}<5$ show discrepancies of about $\pm 20 \%$ when compared to data, with a significant dependency on $\tau_{\perp}$ for some generators, while the prediction power of all generators for final states with $n^{\text {jet }} \geq 5$ lays within $\pm 50 \%$ with less dependency on $\tau_{\perp}$. None of the selected generators gives a satisfactory prediction for the whole evaluation space.

A data-to-MC simulations comparison of the fiducial cross section $\sigma\left(n^{\text {jet }}\right)$ yields reasonably good agreement with the generators for $2 \leq n^{\text {jet }} \leq 3$, but shows significantly disagreement for $n^{\text {jet }}>3$. In addition, the predictions for $\sigma\left(n^{\text {jet }}>3\right)$ show a large spread between generators. For example, SHERPa predicts a $30 \%$ higher cross section than data, while Herwig with the dipole shower and MADGRAPH5_AMC predicts $30 \%$ lower. These differences affect the normalisation of the distributions shown in the left panel of Figure 1. In case of $n^{\mathrm{jet}} \geq 6$ and $\tau_{\perp} \gtrsim 0.1, \Sigma_{\tau_{\perp}}$ predicted by SHERPA is about $50 \%$ larger than for data, with SHERPA predicting a larger normalisation already. The HeRwIg generator configured with the dipole shower, on the other hand, predicts a $50 \%$ lower $\Sigma_{\tau_{\perp}}$ than data in this region, but with about $30 \%$ smaller normalisation.

\section{Measurement of the strong coupling constant}

The evolution of the transverse energy-energy correlations (TEEC) between jets in a multijet final state with a varying hard scale allows measuring the $\alpha_{\mathrm{S}}$ evolution on the same scale. The TEEC is given by

$$
\frac{1}{\sigma} \frac{d \Sigma}{d \cos \phi}=\frac{1}{\sigma} \sum_{i, j} \int d \sigma \frac{E_{\mathrm{T}, i} E_{\mathrm{T}, j}}{\left(E_{\mathrm{T}, i}+E_{\mathrm{T}, j}\right)^{2}} \delta\left(\cos \Delta \varphi_{i j}-\cos \phi\right)
$$



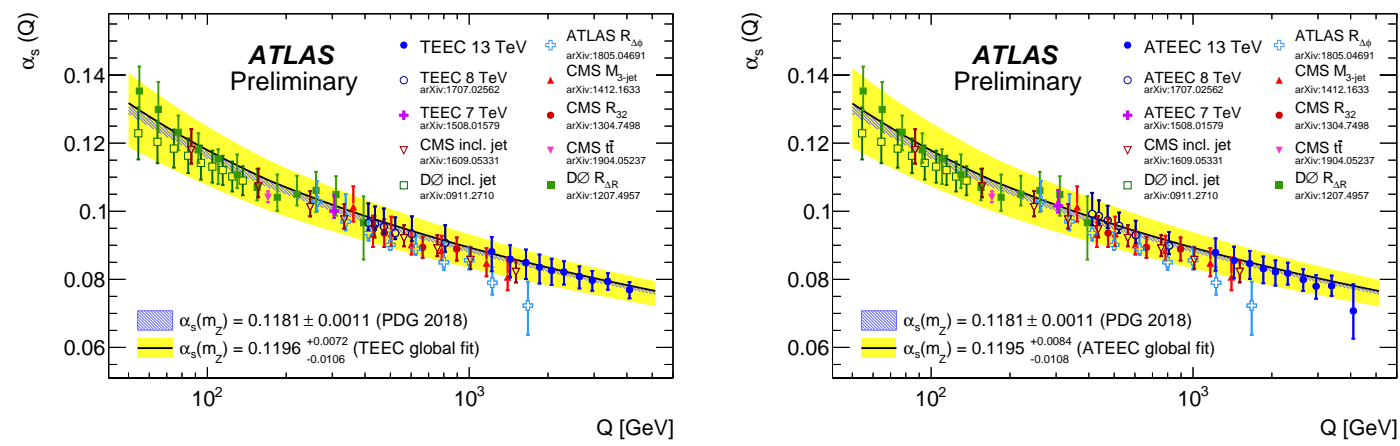

Figure 3: The strong coupling constant $\alpha_{\mathrm{S}}$ as function of the momentum transfer $Q$ for this and previous measurements, extracted from the analysis of TEEC (left) and ATEEC (right) distributions (figures taken from Ref. [3]).

Here $E_{\mathrm{T} . i(j)}$ is the transverse energy of jet $i(j)$, and $\Delta \varphi_{i j}$ is the azimuthal distance between any two jets $i$ and $j$ in the event. The associated azimuthal asymmetries (ATEEC) measures the difference between the forward $(\cos \phi>0)$ and the backward $(\cos \phi<0)$ TEEC,

$$
\frac{1}{\sigma} \frac{d \Sigma^{\mathrm{asymm}}}{d \cos \phi}=\frac{1}{\sigma}\left(\left.\frac{\Sigma}{d \cos \phi}\right|_{\phi}-\left.\frac{\Sigma}{d \cos \phi}\right|_{\pi-\phi}\right) .
$$

The ATLAS measurement of (A)TEEC uses multijet final states with anti- $k_{t}$ jets clustered with $R=0.4$ within $|\eta|<2.4$. Jets are required to have $p_{\mathrm{T}}>60 \mathrm{GeV}$. As for the event shape measurement, the scale evaluating the hardness of the interaction is $H_{\mathrm{T} 2}$. To be considered, each event has to have at least two jets passing the jet selection. In addition, $H_{\mathrm{T} 2}>1 \mathrm{TeV}$ is required for the event to be included in the measurement. The (A)TEEC distributions are measured inclusively and in bins of $H_{\mathrm{T} 2}$. All data distributions are unfolded to particle level.

For the measurement, the strong coupling constant at the $Z$-boson mass pole $\left(\alpha_{\mathrm{S}}\left(m_{Z}\right)\right)$ is determined by fitting theoretical predictions to the inclusive TEEC and ATEEC distributions shown in Figure 2 and the corresponding distributions in $H_{\mathrm{T} 2}$ bins. The evolution of $\alpha_{\mathrm{S}}\left(m_{Z}\right) \rightarrow \alpha_{\mathrm{S}}(Q)$ is performed using the fit results in the $H_{\mathrm{T} 2}$ bins and evolving them by applying NLO solutions to renormalisation group equations. Here $H_{\mathrm{T} 2}$ provides the measure for the momentum transfer $Q$. The resulting running of $\alpha_{\mathrm{S}}$ as function of $Q$ is shown in Figure 3. This measurement does not only show a very good agreement of the $\alpha_{\mathrm{S}}\left(m_{Z}\right)$ determined by a global fit to the (A)TEEC distributions with the world average, as indicated in the figures, but also extends the previous measurements to a multiple $\mathrm{TeV}$ range at high precision.

\section{Conclusion}

The measurement of hadronic event shapes with hard jets, performed using the whole LHC Run 2 dataset collected by the ATLAS experiment, shows several significant shortcomings of hard emission modeling by state-of-the-art generators. This measurement, which now includes events with a large number of hard jets, can provide important input to ongoing an future model and generator developments. The measurement of energy-energy correlations and the corresponding 
azimuthal asymmetry, also using the full Run 2 dataset, provides access to $\alpha_{\mathrm{S}}$ at highest momentum transfer scales. The measured $\alpha_{\mathrm{S}}\left(m_{Z}\right)$ agrees very well with the present world-average value, while the measured running of $\alpha_{\mathrm{S}}$ tests theoretical predictions at highest scales ever.

\section{References}

[1] ATLAS Collaboration, The ATLAS Experiment at the CERN Large Hadron Collider, JINST 3 S08003 (2008)

[2] ATLAS Collaboration, Measurement of hadronic event shapes in multijet final states at $\sqrt{s}=13 \mathrm{TeV}$ with the ATLAS detector, [hep-ex/2007.12600] (2020)

[3] ATLAS Collaboration, Determination of the strong coupling constant and test of asymptotic freedom from Transverse Energy-Energy Correlations in multijet events at $\sqrt{s}=13 \mathrm{TeV}$ with the ATLAS detector, ATLAS-CONF-2020-025 http://cds.cern.ch/record/2725553 (2020)

[4] Cacciari, M., Salam, G.P. and Soyez, G., The anti- $k_{t}$ jet clustering algorithm, JHEP 04063 (2008) [hep-ph/0802.1189]

[5] Cacciari, M.,Salam, G.P. and Soyez, G., FastJet User Manual, Eur. Phys. J. C 721896 (2012) [hep-ph/1111.6097]

[6] ATLAS Collaboration, Jet reconstruction and performance using particle flow with the ATLAS Detector, Eur. Phys. J. C 77466 (2017) [hep-ex/1703. 10485]

[7] ATLAS Collaboration, Jet energy scale and resolution measured in proton-proton collisions at $\sqrt{s}=13 \mathrm{TeV}$ with the ATLAS detector, [hep-ex/2007.02645] (2020)

[8] Banfi, A., Salam, G.P. and Zanderighi, G., Phenomenology of event shapes at hadron colliders, JHEP 06038 (2010) [hep-ph/1001. 4082]

[9] Banfi, A., Salam, G.P. and Zanderighi, G., Resummed event shapes at hadron - hadron colliders, JHEP 08062 (2004) [hep-ph/0407287]

[10] Sjöstrand, T. et al., An introduction to PYTHIA 8.2, Comput. Phys. Commun. 191 159-177 (2015) [hep-ph/1410.3012]

[11] Gleisberg, T. et al., Event generation with SHERPA 1.1, JHEP 02007 (2009) [hep-ph/0811.4622]

[12] Bellm, J. et al., Herwig 7.0/Herwig++ 3.0 release note, Eur. Phys. J. C. 76 4, 196 (2016) [hep-ph/1512.01178]

[13] Bellm, J. et al., Herwig 7.2 release note, Eur. Phys. J. C. 80 5, 452 (2019) [hep-ph/1912.96509]

[14] Alwall, J. et al., The automated computation of tree-level and next-to-leading order differential cross sections, and their matching to parton shower simulations, JHEP 07079 (2014) [hep-ph/1405.0301] 